\title{
PROCESSAMENTO DE SINAIS ULTRASSÔNICOS PARA A CARACTERIZAÇÃO DE UM AÇO AUSTENÍTICO RESISTENTE AO CALOR COM DIFERENTES ESTADOS DE ENVELHECIMENTO*
}

Natalie Chaves de Siqueira
Mónica Patrícia Arenas
Priscila Duarte de Almeida $^{3}$
Leonardo Sales de Araújo $^{4}$
Carlos Bruno Eckstein
Luiz Henrique de Almeida
Laudemiro Nogueira Júnior $^{7}$
Gabriela Ribeiro Pereira $^{8}$

Resumo

Aços inoxidáveis austeníticos fundidos por centrifugação, da classe HP, são comumente utilizados na indústria de óleo e gás para a fabricação de tubos usados em fornos de reforma e pirólise. Essas ligas possuem uma elevada resistência à oxidação, elevada estabilidade térmica e elevada resistência à fluência em altas temperaturas, sendo esses requisitos necessários para aplicação nos fornos. Mudanças microestruturais, conhecidas como envelhecimento, podem ocorrer durante a operação, influenciado na resistência à fluência. Desta forma, a caracterização dos estados de envelhecimento de uma liga HP torna-se necessária para avaliar a sua vida remanescente. O objetivo deste estudo é caracterizar duas condições microestruturais de uma liga HP com a microscopia eletrônica de varredura e o ensaio não destrutivo de ultrassom. O processamento do sinal ultrassônico, utilizando a Transformada Rápida de Fourier (Fast Fourier Transform - FFT), foi realizado sobre o segundo eco de fundo do sinal ultrassônico, permitindo correlacionar a análise espectral do sinal com a microestrutura do aço HP. De acordo com a metodologia proposta, os dois estados de envelhecimento foram caracterizados através do processamento do sinal ultrassônico.

Palavras-chave: Aço Austenítico; Envelhecimento; Ensaio Ultrassônico; Análise Espectral

\section{ULTRASONIC SIGNAL PROCESSING FOR THE CHARACTERIZATION OF A HEAT-RESISTANT AUSTENITIC STEEL WITH DIFFERENTES AGING STATES}

\section{Abstract}

HP austenitic stainless alloys are extensively applied in oil and gas industry as tubes used in reformer and pyrolysis furnaces. These steels exhibit high resistance to oxidation at high temperatures, thermal stability and resistance to creep. Microstructural changes, known as aging, occur during service in harsh operational conditions, leading to a decrease in creep resistance. Therefore, the characterization of the different states of aging of a HP steel is necessary for the life assessment. This study aims to characterize two different microstructural conditions of a modified HP steel via scanning electron microscopy and ultrasonic testing. The Fast Fourier Transform (FFT) was performed in the second back wall echo of the acquired ultrasonic signal. The spectral analysis allowed to establish a correlation between the microstructure and signal response. According to the developed methodology, two different states of aging were successfully characterized through the ultrasonic signals processing.

Keywords: Austenitic steels; Aging; Ultrasonic testing; Spectral analysis. 
1 Engenheira de Materiais, M.Sc., doutoranda, Departamento de Engenharia Metalúrgica e de Materiais/UFRJ-COPPE, LNDC-Laboratório de Ensaios Não Destrituivos Corrosão e Soldagem, Rio de Janeiro, Rio de Janeiro, Brasil.

2 Engenheira Física, M.Sc., doutoranda, Departamento de Engenharia Metalúrgica e de Materiais/UFRJ-COPPE, LNDC-Laboratório de Ensaios Não Destrituivos Corrosão e Soldagem, Rio de Janeiro, Rio de Janeiro, Brasil.

3 Engenheira de Materiais, M.Sc., Engenheira Pesquisadora, Departamento de Engenharia Metalúrgica e de Materiais/UFRJ-COPPE, LNDC-Laboratório de Ensaios Não Destrituivos Corrosão e Soldagem, Rio de Janeiro, Rio de Janeiro, Brasil.

4 Engenheiro Metalúrgico, D.Sc., Professor Adjunto, Departamento de Engenharia Metalúrgica e de Materiais/UFRJ-COPPE, Universidade Federal do Rio de Janeiro-UFRJ, Rio de Janeiro, Rio de Janeiro e Brasil.

5 Engenheiro Metalúrgico, D.Sc., Consultor Sênior, CENPES/PETROBRAS, Petróleo Brasileiro S.A. - PETROBRAS, Rio de Janeiro, Rio de Janeiro, Brasil.

6 Engenheiro Metalúrgico, D.Sc., Professor Titular, Departamento de Engenharia Metalúrgica e de Materiais, Universidade Federal do Rio de Janeiro-UFRJ, Rio de Janeiro, Rio de Janeiro e Brasil.

7 Engenheiro Mecânico, D.Sc., Consultor Sênior, CENPES/PETROBRAS, Petróleo Brasileiro S.A. PETROBRAS, Rio de Janeiro, Rio de Janeiro, Brasil.

8 Física, D.Sc., Professora Adjunta, Departamento de Engenharia Metalúrgica e de Materiais, Universidade Federal do Rio de Janeiro-UFRJ, Rio de Janeiro, Rio de Janeiro e Brasil. 


\section{INTRODUÇÃO}

Aços inoxidáveis austeníticos resistentes ao calor, da classe HP, são frequentemente usados nas indústrias petroquímica para a fabricação de tubos de reforma e pirólise. Esses tubos são projetados para operar durante $100.000 \mathrm{~h}$ em condições extremas de temperatura e pressão, como recomendado pela API [1,2]. As ligas HP apresentam vantagens quando comparados às super ligas de níquel, como preço competitivo, resistência à oxidação em altas temperaturas e elevada resistência à fluência (3). A adição de elementos como $\mathrm{Nb}$ e Ti melhoram a estabilidade microestrutural e assim suas propriedades mecânicas (4). A composição destas ligas segue a especificação da ASTM A297 (5).

Os tubos de reforma são submetidos a diferentes temperaturas ao longo de seu comprimento, ocasionando em mudanças microestruturais (1). Um estudo mais detalhado sobre o perfil de temperatura ao longo dos tubos de reforma foi realizado por Le May et al. (1) onde cada região do tubo é sujeita a diferentes temperaturas recebendo uma classificação própria (1). Na condição as-cast, o aço HP modificado ao Nióbio (HP-Nb) possui uma microestrutura estável formada por uma matriz completamente austenítica, com uma complexa precipitação primária de carbetos nos espaços interdentríticos. A microestrutura é estável para temperaturas abaixo de $650^{\circ} \mathrm{C}$. Para temperaturas acima de $650^{\circ} \mathrm{C}$, o material sofre envelhecimento, caracterizado pelo coalescimento dos carbetos primários e uma extensiva precipitação secundária dos carbetos de cromo $\mathrm{M}_{23} \mathrm{C}_{6}(6,7)$. Em temperaturas acima de $700{ }^{\circ} \mathrm{C}$, os carbetos de nióbio iniciam uma transformação in situ de uma fase conhecida como fase $\mathrm{G}, \mathrm{Ni}_{16} \mathrm{Nb}_{6} \mathrm{Si}_{7}(4,6,8)$. A formação de vazios de fluência ocorre preferencialmente entre a fase $\mathrm{G}$ e a matriz austenítica. Quando estes vazios se interligam podem ocorrer falhas por fluência, um dos mais significativos tipos de danos nesses tubos $(1,3,4,9)$. O ensaio não destrutivo de ultrassom (UT) é uma técnica muito difundida na detecção de defeitos e descontinuidades. Essa técnica também tem sido empregada na avaliação das mudanças microestruturais, uma vez que a propagação da onda ultrassônica nos materiais é significativamente afetada pela variação da microestrutura. Parâmetros como tenacidade à fratura, limite de escoamento, tamanho de grão, velocidade ultrassônica, atenuação e outros podem ser obtidos (1014). Os sinais ultrassônicos são geralmente apresentados no domínio do tempo, contudo, análises mais complexas como avaliação das mudanças microestruturais podem ser extraídas dos sinais no domínio da frequência (11). A análise espectral e a transformada discreta de Fourier (Discrete Fourier Transform - DFT) tem sido largamente utilizada em sinais ultrassônicos para caracterização de materiais $(11,15-$ 18). Kumar et al. (17) correlacionaram as mudanças microestruturais de um aço inoxidável austenítico com a análise espectral. Esta análise ocorreu no primeiro eco de fundo do sinal ultrassônico encontrando uma relação inversamente proporcional entre tamanho de grão e limite de escoamento. Foi observado que o pico de frequência e a largura máxima a meia altura (full width at half maximum - FWHM) diminuíam com o aumento do tamanho de grão. Em outro estudo, Kumar et al. (18) caracterizaram via UT uma liga de $9 \mathrm{Cr}-1$ Mo que sofreu diferentes tratamentos térmicos, observando que com o aumento da temperatura de tratamento a velocidade sônica diminuía devido ao aumento na quantidade de martensita. Assim sendo fica evidente que a técnica de UT é sensível na detecção de variações microestruturais através da análise espectral. Desta maneira, o presente estudo tem como objetivo caracterizar dois estados de envelhecimento de uma liga de aço HP modificado ao Nióbio correlacionando microscopia eletrônica de varredura (MEV) e o ensaio não 
destrutivo de ultrassom. Os sinais ultrassônicos foram analisados no domínio do tempo e da frequência.

\section{MATERIAIS E MÉTODOS}

Foram extraídas duas amostras de um tubo de reforma que esteve em serviço durante 70.000 horas. O diâmetro interno e a espessura do tubo foram de $112 \mathrm{~mm}$ e $11,50 \mathrm{~mm}$, respectivamente. As amostras estiveram expostas a diferentes temperaturas de operação exibindo diferentes estados de envelhecimento, conforme observado na Tabela 1.

Tabela 1. Características das amostras analisadas.

\begin{tabular}{ccc}
\hline Amostra & $\begin{array}{c}\text { Estado de } \\
\text { envelhecimento }\end{array}$ & $\begin{array}{c}\text { Temperatura de } \\
\text { operação }\end{array}$ \\
\hline $\mathrm{A}$ & $\mathrm{IV}$ & $800-900^{\circ} \mathrm{C}$ \\
$\mathrm{B}$ & $\mathrm{V}$ & $900-10000^{\circ} \mathrm{C}$ \\
\hline
\end{tabular}

Foi realizada uma análise química numa amostra com condição as-cast, cuja composição química é apresentada Tabela 2.

Tabela 2: Composição química do aço HP-Nb na condição as-cast.

\begin{tabular}{ccccccccccc}
\hline Elemento & $\mathbf{C}$ & $\mathbf{C r}$ & $\mathbf{N i}$ & $\mathbf{N b}$ & $\mathbf{T i}$ & $\mathbf{M n}$ & $\mathbf{S i}$ & $\mathbf{P}$ & $\mathbf{S}$ & $\mathbf{M o}$ \\
\hline $\begin{array}{c}\text { Composição } \\
\text { [\% wt] }\end{array}$ & 0.45 & 27.0 & 34.6 & 0.74 & 0.05 & 0.91 & 1.24 & 0.021 & 0.010 & 0.031 \\
\hline$\% \mathrm{Fe} \mathrm{Bal.}$ & & & & & & & & & &
\end{tabular}
$\%$ Fe Bal.

\subsection{Microscopia eletrônica de varredura (MEV)}

De cada estado de envelhecimento foram extraídas amostras metalográficas para a análise microestrutural. A caracterização foi realizada usando o microscópio eletrônico de varredura VEGA3 TESCAN com voltagem de $30 \mathrm{kV}$. A análise foi realizada no modo elétrons retroespalhados (EBS) para identificar as fases presentes nas amostras. De cada amostra foram adquiridas 30 imagens, no modo EBS, para identificar as diferentes fases microestruturais. Posteriormente, foi utilizado o software de domínio público ImageJ® para o processamento e quantificação dos carbetos de cromo e de nióbio, assim como a contagem dos vazios de fluência (19-21).

\subsection{Ensaio de ultrassom}

O ensaio não destrutivo de ultrassom foi realizado utilizando o modo pulso-eco com equipamento Sonotron Isonic 2005, transdutor não focalizado de frequência central de 1,6 MHz e acoplamento realizado com mel. Para aquisição dos sinais foi utilizado um osciloscópio (Tecktronix MSO 4034) com frequência de amostragem de $250 \mathrm{MA} / \mathrm{s}$. Em cada uma das amostras foram adquiridos 30 sinais ultrassônicos sobre uma área de $50 \mathrm{~mm} \times 120 \mathrm{~mm}$. Este procedimento foi realizado seis vezes com o objetivo de garantir confiabilidade nos resultados e validar a metodologia estabelecida. Os dados coletados foram processados em uma rotina Matlab®. 


\subsection{Processamento dos sinais}

Os sinais ultrassônicos foram analisados tanto no domínio do tempo como da frequência. Para o primeiro caso, foi calculada a média dos sinais utilizando a correlação cruzada para fazer a correção da defasagem entre os sinais (22). Com o intuito de verificar a validade das análises efetuadas no domínio da frequência, optouse em analisar a média dos sinais para 5, 10, 20 e 30 sinais. Na rotina de processamento foi realizado a remoção do sinal DC contido no sinal de input, seguido da aplicação da FFT entre os dois ecos de fundo consecutivos do sinal $A$-scan para os 4 estudos.

\section{RESULTADOS E DISCUSSÃO}

\subsection{Microscopia eletrônica de varredura}

A condição as-cast de um aço HP, modifcado ao $\mathrm{Nb}$, caracteriza-se pela presença de carbetos primários $\mathrm{M}_{7} \mathrm{C}_{3}$ e/ou $\mathrm{M}_{23} \mathrm{C}_{6}$, ricos em $\mathrm{Cr}$, e $\mathrm{MC}$, ricos em $\mathrm{Nb}$. O fenômeno de envelhecimento inicia-se quando o material é exposto em temperaturas acima de $650^{\circ} \mathrm{C}$. Assim sendo, a amostra A, exposta a temperaturas entre $800^{\circ} \mathrm{C}$ e $900^{\circ} \mathrm{C}$, exibe o estado de envelhecimento IV. Na Figura 1.a observa-se que carbetos primários ricos em cromo assumem forma de blocos, os carbetos $\mathrm{NbC}$ sofrem uma transformação parcial para fase $\mathrm{G}$ e a precipitação secundária de carbetos de cromo ocorrem tanto na região interdentrítica como intradendrítica da matriz. Por outro lado, a amostra $B$, com estado de envelhecimento $V$, apresenta uma transformação quase completa do $\mathrm{NbC}$ para a fase $\mathrm{G}$, observa-se também a formação e o alinhamento de vazios de fluência (regiões mais escuras), Figura 1.b.

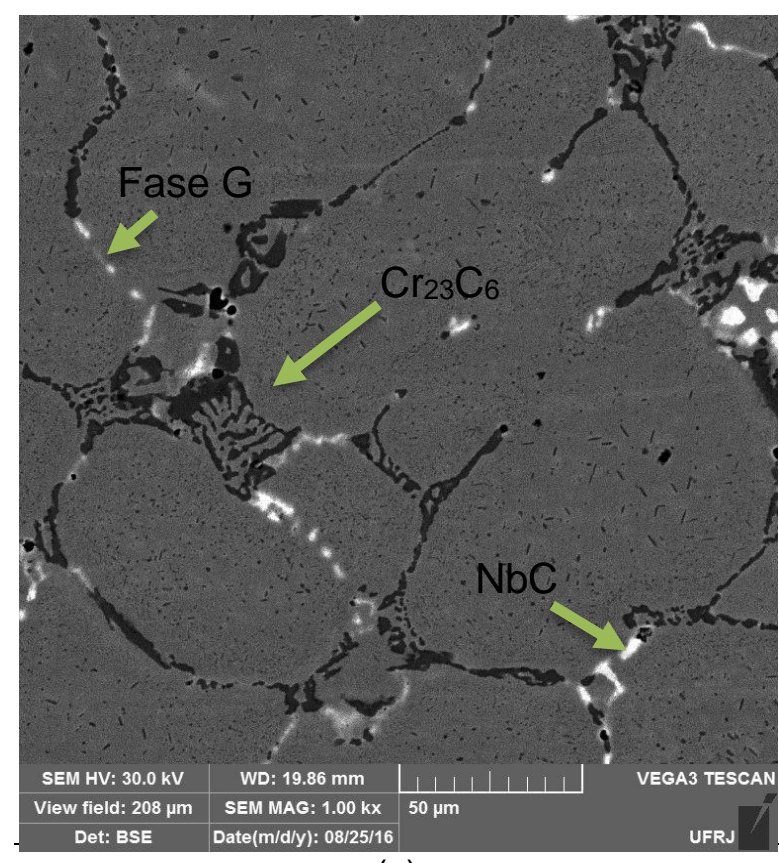

(a)

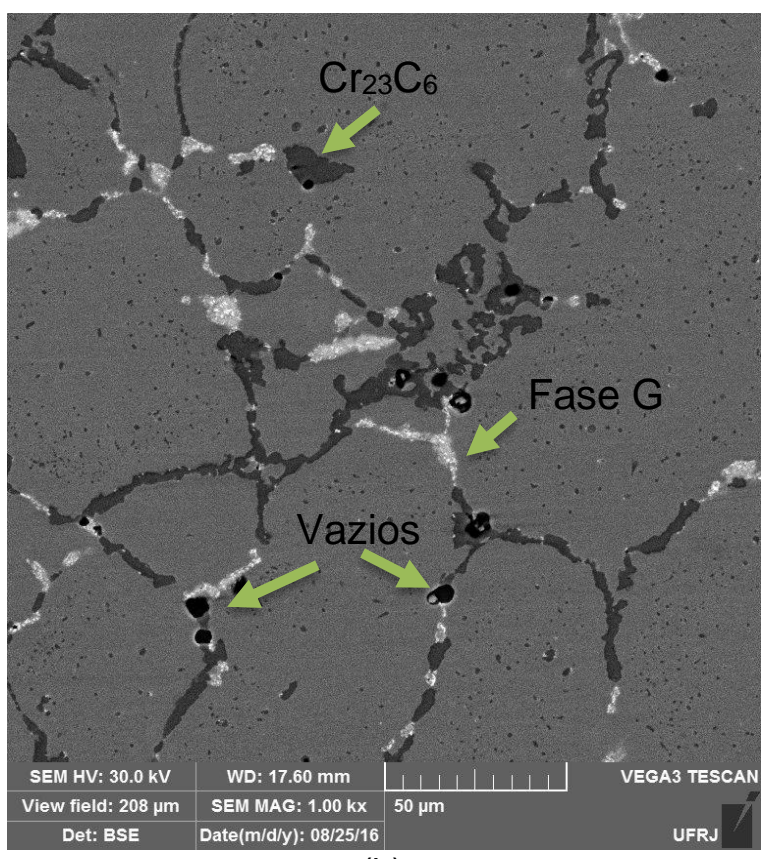

(b)

Figura 1. Micrografia MEV no modo elétrons retroespalhados nas amostras com estados de envelhecimento: (a) IV e (b) V.

Para cada estado de envelhecimento foi realizado um processamento de imagens nas micrografias obtidas. Isto com o objetivo de realçar o contraste das diferentes fases 
microestruturais e assim realizar a contagem da fração volumétrica baseado na norma ASTM E1382 (21). A fração volumétrica dos carbetos NbC e fase $\mathrm{G}$ foi contabilizada em conjunto devido à similaridade do contraste entre ambas as fases. A fração volumétrica de carbetos ricos em $\mathrm{Cr}$ e de vazios foi contabilizada separadamente devido ao contraste bem definido. A fração volumétrica das microestruturas é apresentado na Figura 2. Verifica-se que a fração volumétrica de vazios aumenta em função do estado de envelhecimento, assim como a fração de carbetos, sendo maior a porcentagem para o carbetos de cromo.

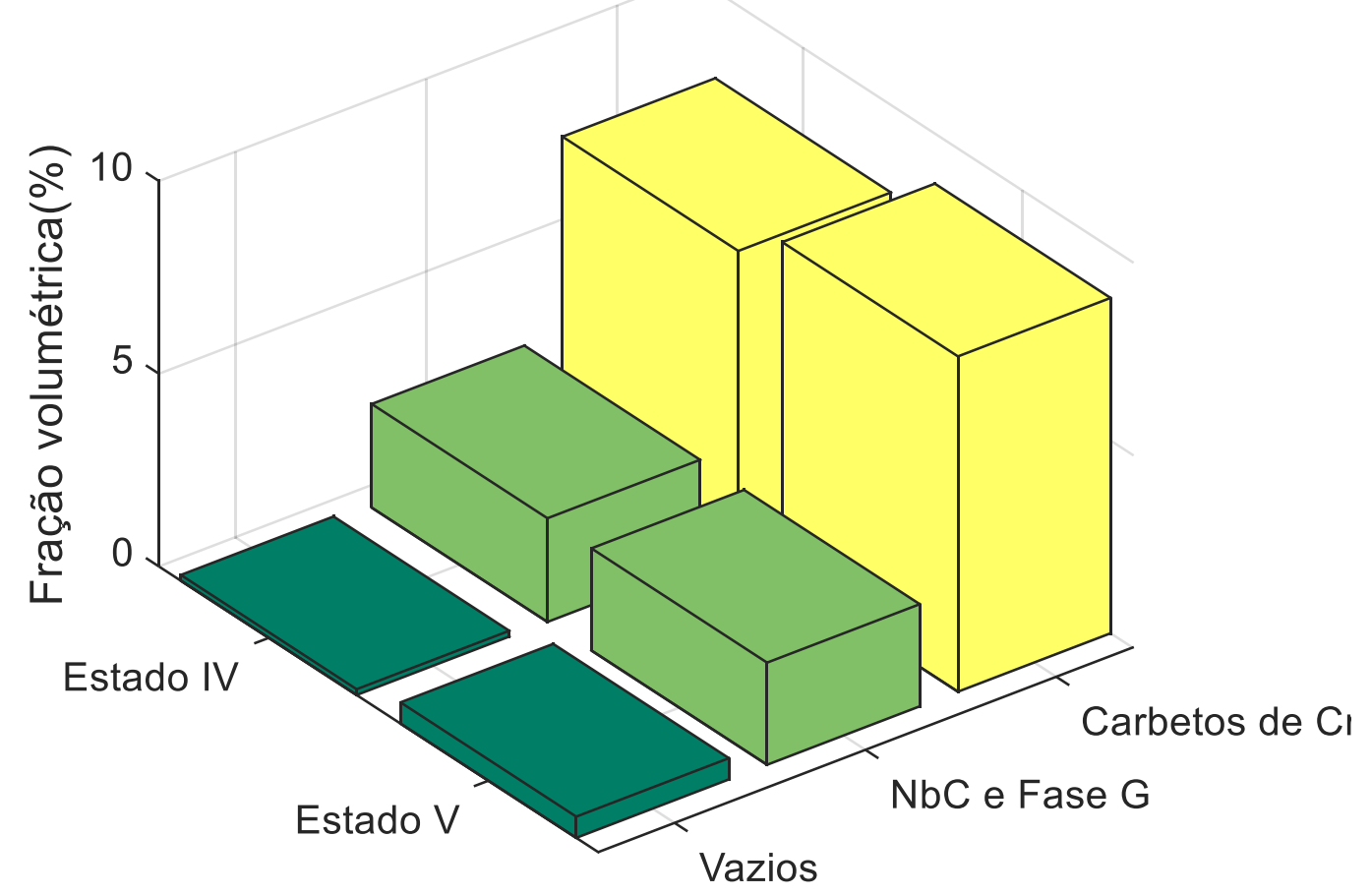

Figura 2. Fração volumétrica das fases presentes para cada uma das amostras analisadas.

\subsection{Processamento dos sinais ultrassônicos}

A seguir é apresentado a metodologia desenvolvida para a correição do atraso dos sinais A-scan. Foram adquiridos 30 sinais ultrassônicos na amostra com estado de envelhecimento $\mathrm{V}$, os quais foram plotados em um mesmo gráfico no domínio do tempo. Verifica-se na Figura 3.a a presença do atraso entre os sinais, sendo necessário realizar a correlação cruzada dos sinais, Figura 3.b. Verificando e corrigindo o atraso dos sinais foi gerado um novo gráfico A-scan com a média dos sinais, observado na Figura 3.c. 


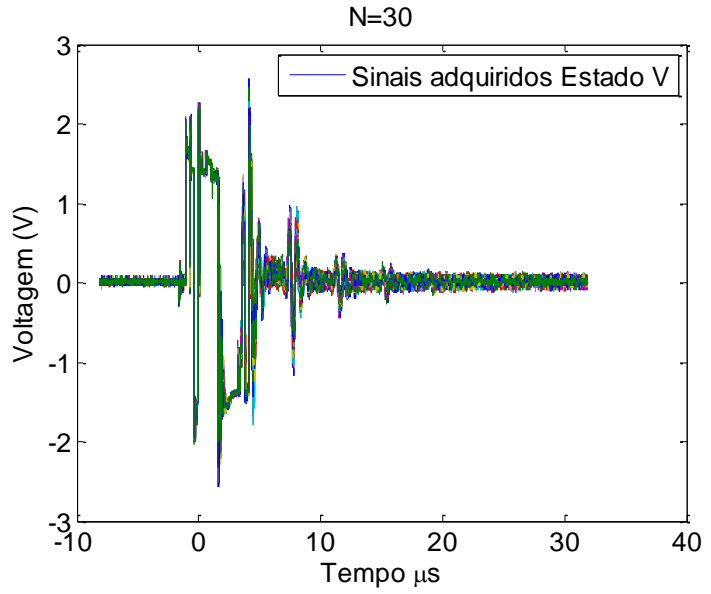

(a) A-scan

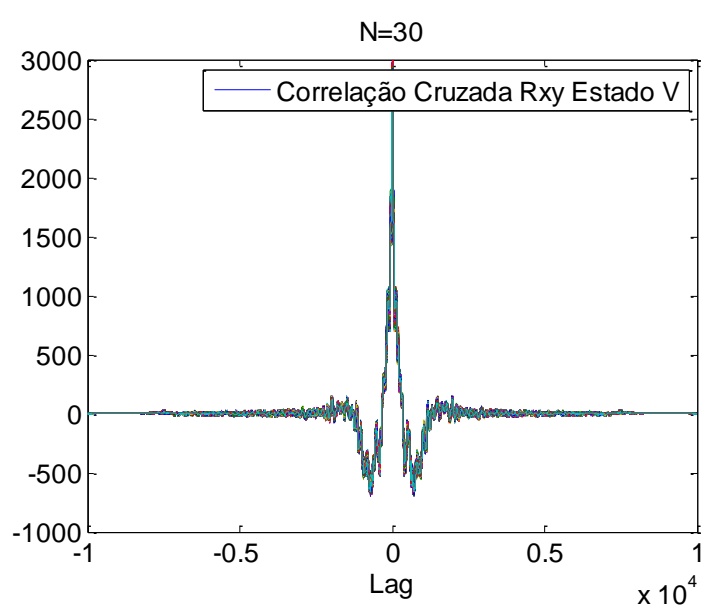

(b) $R_{x y}$ para $\mathrm{N}=30$ sinais

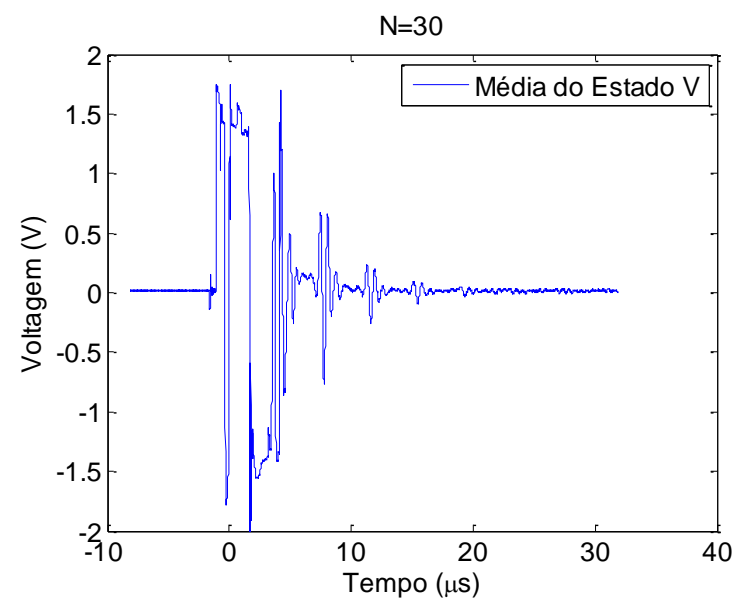

(c) Média dos 30 sinais após o processamento do sinal

Figura 3. Demonstração das etapas do processamento de sinais ultrassônicos em amostras HP-Nb para o estado de envelhecimento V. (a) Sinais A-scan, (b) correlação cruzada dos sinais e (c) média dos sinais após correção da defasagem.

\subsection{Análise espectral}

A análise espectral, variando a quantidade de sinais médios, pode ser observada na Figura 4. Aumentando a quantidade de sinais, acima de 20, há uma distinção maior entre os estados IV e V, indicando uma maior eficiência para essa quantidade de sinais. Observa-se em todos os gráficos um pico na frequência de $1,465 \mathrm{MHz}$, próximo à largura de banda do transdutor. Verifica-se que a amplitude espectral é influenciada diretamente pela condição microestrutural do material. Observa-se que a amostra com estado de envelhecimento IV tem uma maior amplitude se comparado com o estado de envelhecimento $V$. Este comportamento pode estar associado à fração volumétrica dos vazios de fluência, pois no estado $V$ esta porcentagem é maior, atenuando o sinal ultrassônico. 


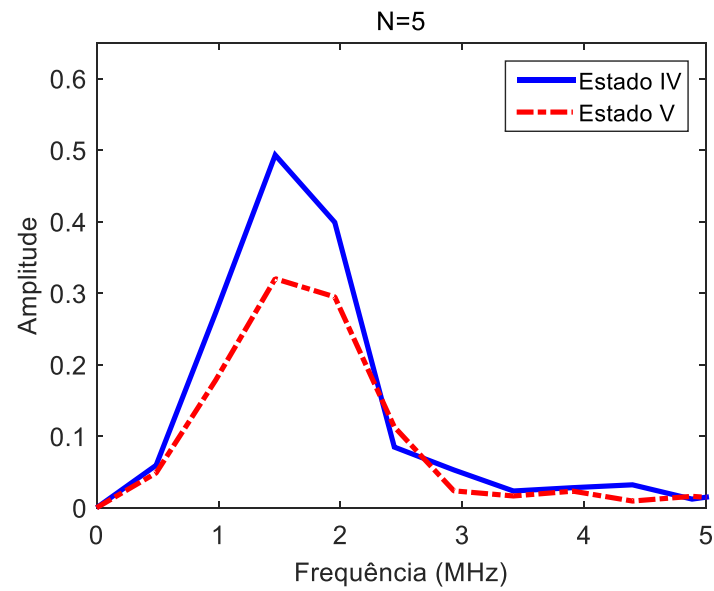

(a)

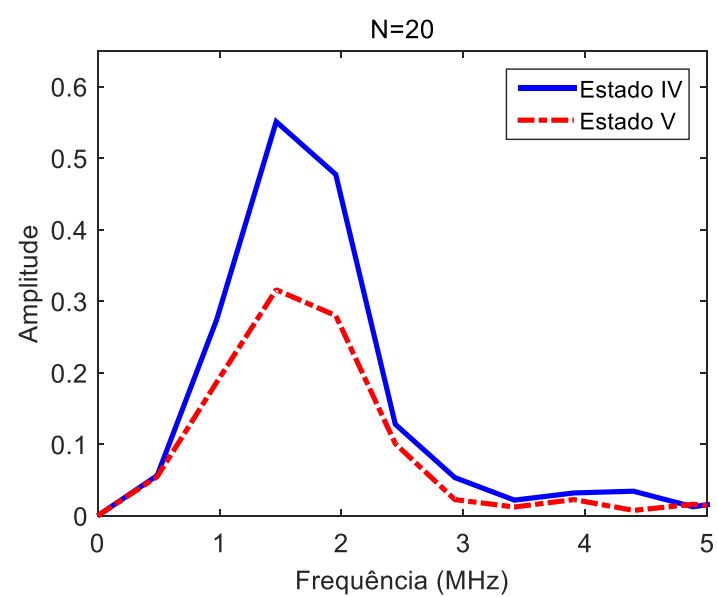

(c)

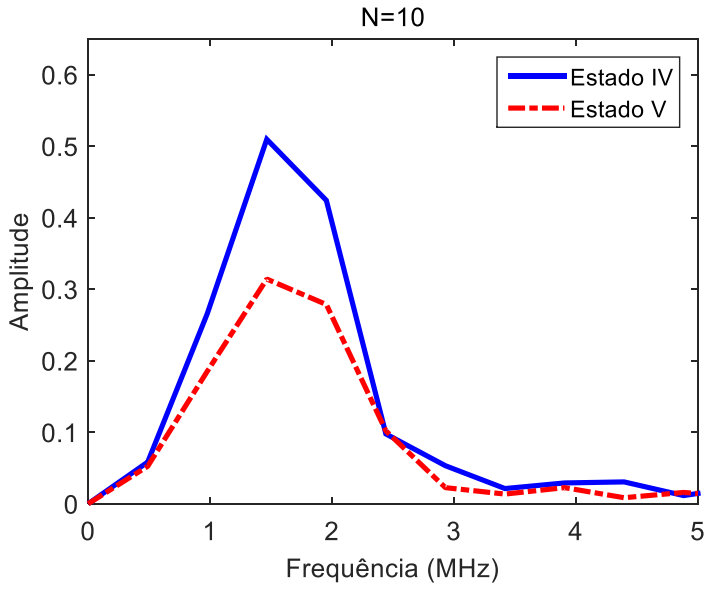

(b)

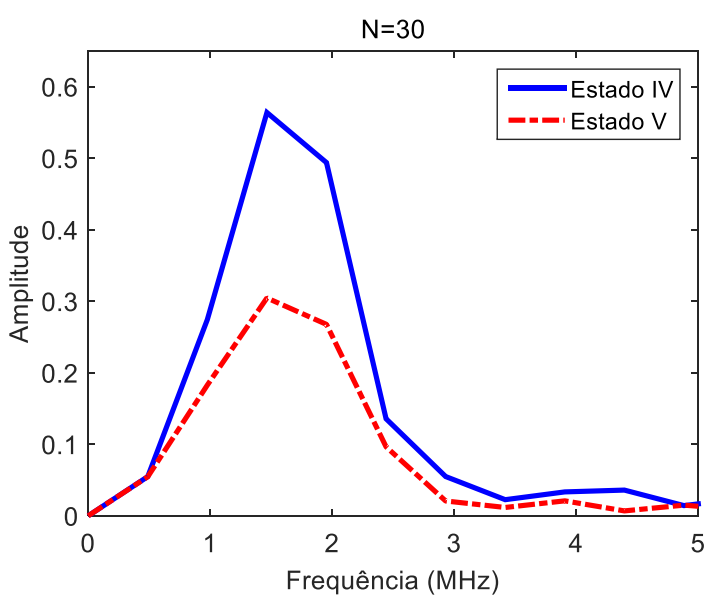

(d)

Figura 3. Sinal espectral do segundo eco de fundo nas amostras HP modificadas ao Nb. Observa-se uma maior amplitude para a amostra com estado IV e uma maior atenuação do sinal para o estado $\mathrm{V}$ em todas as abordagens. (a) $\mathrm{N}=5$, (b) $\mathrm{N}=10$, (c) $\mathrm{N}=20$ e (d) $\mathrm{N}=30$.

\section{CONCLUSÃo}

O ensaio não destrutivo de ultrassom realizado em amostras HP, modificado ao nióbio, demonstrou uma boa correlação entre a resposta do sinal ultrassônico e a variação microestrutural decorrente do envelhecimento. A aplicação da FFT sobre o segundo eco de fundo demonstrou que a análise espectral do sinal ultrassônico é muito sensível à presença de vazios de fluência, ocorrendo diminuição da amplitude espectral com aumento na fração volumétrica de vazios.

\section{Agradecimentos}

À equipe do Laboratório de Ensaios Não Destrutivos, Corrosão e Soldagem LNDC/COPPE/UFRJ. Ao CNPq, CAPES e FAPERJ pelo suporte financeiro. À Petrobras pelo fornecimento das amostras. 


\section{REFERÊNCIAS}

1. Le May I, da Silveira TL, Vianna $\mathrm{CH}$. Criteria for the evaluation of damage and remaining life in reformer furnace tubes. Int $\mathrm{J}$ Press Vessel Pip [Internet]. 1996;66(1-3):233-41. Available from:

http://www.sciencedirect.com/science/article/pii/0308016195000984

2. API-530. Calculation of Heater-Tube Thickness in Petroleum Refineries Calculation of Heater-Tube Thickness in Petroleum Refineries. October. Washington D.C.: American Petroleum Institute; 1996.

3. de Almeida LH, Ribeiro AF, Le May I. Microstructural characterization of modified $25 \mathrm{Cr}-35 \mathrm{Ni}$ centrifugally cast steel furnace tubes. Mater Charact. 2003;49(3):219-29.

4. Nunes FC, de Almeida LH, Dille J, Delplancke JL, Le May I. Microstructural changes caused by yttrium addition to NbTi-modified centrifugally cast HP-type stainless steels. Mater Charact. 2007;58(2):132-42.

5. ASTM-A297/A297M. Standard Specification for Castings, Iron-Chromium, IronChromium-Nickel, Corrosion Resistant, for General Application. Standard ASTM ASTM International; 2008 p. 1-3.

6. Barbabela GD, de Almeida LH, da Silveira TL, Le May I. Phase characterization in two centrifugally cast HK stainless steel tubes. Mater Charact. 1991 Jan;26(1):1-7.

7. Barbabela GD, Almeida LH De, Luiz T, Le May I. Role of Nb in modifying the microstructure of heat-resistant cast HP steel. Mater Charact. 1991;26(3):1937.

8. Pedro Ibañez RA, de Almeida Soares GD, de Almeida LH, Le May I. Effects of Si content on the microstructure of modified-HP austenitic steels. Mater Charact. 1993;30(4):243-9.

9. Sposito G, Ward C, Cawley P, Nagy PB, Scruby C. A review of non-destructive techniques for the detection of creep damage in power plant steels. NDT E Int [Internet]. 2010;43(7):555-67. Available from: http://dx.doi.org/10.1016/j.ndteint.2010.05.012

10. Thompson RB. Ultrasonic measurement of mechanical properties. In: IEEE Ultrasonics Symposium [Internet]. 1996. p. 735-44. Available from: http://ieeexplore.ieee.org/lpdocs/epic03/wrapper.htm?arnumber $=584079$

11. Krüguer SE, Rebello JMA, Camargo PC De. Hydrogen damage detection by ultrasonic spectral analysis. NDT E Int. 1999;32:275-81.

12. Nanekar PP, Shah BK. Characterization of material properties by ultrasonics. Vol. II, Word Congress on Engineering. BARC Newsletter; 2009. p. 1538-44.

13. Vary A. Ultrasonic testing. In: Workman, G.L.,. Kishoni, D., Moore P., editor. Non-destructive Testing Handbook. 3rd ed. Ohio, USA: ASNT Handbook; 2007. p. 588.

14. Palanichamy $P$, Joseph $A$, Jayakumar $T$, Raj $B$. Ultrasonic velocity measurements for estimation of grain size in austenitic stainless steel. NDT Int [Internet]. 1995;28(3):179-85. Available from: http://linkinghub.elsevier.com/retrieve/pii/096386959500011L

15. Kumar A, Jayakumar T, Palanichamy P, Raj B. Influence of grain size on ultrasonic spectral parameters in AISI type 316 stainless steel. Scr Mater [Internet]. 1999;40(3):333-40. Available from: http://linkinghub.elsevier.com/retrieve/pii/S1359646298004357

16. Stella J, Cerezo J, Rodríguez E. Characterization of the sensitization degree in 
the AISI 304 stainless steel using spectral analysis and conventional ultrasonic techniques. NDT E Int. 2009;42(4):267-74.

17. Kumar A, Jayakumar $T$, Raj B. Ultrasonic spectral analysis for microstructural characterization of austenitic and ferritic steels. Philos Mag A [Internet]. 2000 Nov [cited 2017 Jan 30];80(11):2469-87. Available from: http://www.tandfonline.com/doi/abs/10.1080/01418610008216486

18. Kumar A, Laha K, Jayakumar T, Rao KBS, Raj B. Comprehensive microstructural characterization in modified $9 \mathrm{Cr}-1$ Mo ferritic steel by ultrasonic measurements. Metall Mater Trans A [Internet]. 2002 Jun [cited $2016 \mathrm{Nov}$ 8];33(6):1617-26. Available from: http://link.springer.com/10.1007/s11661-0020171-9

19. Rasband WS. ImageJ [Internet]. National Institutes of Health. Bethesda, Maryland, USA; [cited 2016 Nov 8]. Available from:

https://imagej.nih.gov/ij/index.html

20. Lombardo S, Costa FH, Hashimoto TM, Pereira MS, Abdalla AJ. Method for the calculation of volumetric fraction of retained austenite through the software for analysis of digital images. $19^{\circ}$ Congresso Brasileiro de Engenharia e Ciência dos Materiais - CBECiMat, 21 a 25 de novembro de 2010, Campos do Jordão, SP, Brasil MÉTODO; 2010.

21. ASTM E1382. Standard test methods for determining average grain size using semiautomatic and automatic image analysis. American Society for Testing and Materials; 1997.

22. Oppenheim A V., Alan S. Willsky. Signals \& Systems. Boston: Massachusetts Institute of Technology;

23. ASTM E494-10. Standard practice for measuring ultrasonic velocity in materials. Annual Book of ASTM Standards. PA, United States: ASTM International; 2011. p. 1-15.

24. Filho JSC, Baroni DB, Lamy CA, Bittencourt MSQ, Motta MS, Su J. Neural network to measure volumetric fraction of a bubbly column using ultrasonic wave. In: COBEM 2009. Gramada, RS, Brazil: 20th International Congress of Mechanical Engineering; 2009. 